\title{
1THz-Bandwidth Polarization-Diverse Optical Phase Conjugation of 10x114Gb/s DP-QPSK WDM Signals
}

\author{
M.F.C. Stephens*, M. Tan, I.D. Phillips, S. Sygletos, P. Harper, N.J. Doran \\ Aston Institute of Photonic Technologies, Aston University, Aston Triangle, Birmingham B4 7ET, UK \\ *m.stephens@aston.ac.uk
}

\begin{abstract}
Polarization diverse optical phase conjugation of a 1THz spectral-band 1.14Tb/s DP-QPSK WDM multiplex is demonstrated for the first time, showing a worst case $\mathrm{Q}^{2}$ penalty of $0.9 \mathrm{~dB}$ over all conjugate wavelengths, polarizations and OSNR.

OCIS codes: (060.4370) Nonlinear optics, fibers, (190.4380) Nonlinear optics, four-wave mixing, (070.5040) Phase conjugation
\end{abstract}

\section{Introduction}

The nonlinear process of optical phase conjugation (OPC), whereby the frequency spectrum of a signal is mirrored in real-time via creation of a conjugate, has historically been considered in telecoms for dispersion compensation via mid-span spectral inversion (MSSI) [1]. OPC nonlinear elements, typically operating via $\chi^{2}$ or $\chi^{3}$ susceptibility have ranged from discrete semiconductor optical amplifiers/lasers [2] to periodically poled lithium niobate crystals (PPLN) [3] and optical fibers such as highly nonlinear fiber (HNLF) [4]. More recently with DSP algorithms compensating for linear impairments in coherent receivers, OPC is being re-examined as a potentially efficient technique for parallel compensation of multichannel nonlinear transmission impairments [5].

A practical conjugator should offer input polarization diversity to avoid the need for precise polarization alignment of pump and signal, and hence must show compatibility with polarization multiplexed (PM) signals such as the semi-standardized dual-polarization (DP)-QPSK format. Polarization diversity can be realized by placing the nonlinear element in a loop, thus propagating orthogonal components of pump and signals bidirectionally [4]. For this technique to be cost effective it is important that the OPC operates over a wide spectral bandwidth to minimize the number of pump wavelengths/OPC bands required to cover the full transmission spectral bandwidth. Although impressive recent progress has been reported demonstrating conjugation of a narrowband ( 150GHz) PM-OFDM 'superchannel' [6], there has been no demonstration of a wide bandwidth, polarization diverse WDM OPC with numerous PM signals and coherent detection. Our results demonstrate an OPC capable of conjugating $>1 \mathrm{THz}$ optical bandwidth and for the first time conjugation of a $1.14 \mathrm{~Tb} / \mathrm{s}(10 \mathrm{x} 114 \mathrm{~Gb} / \mathrm{s})$ multiplex of DP-QPSK signals.

\section{Experimental set-up}

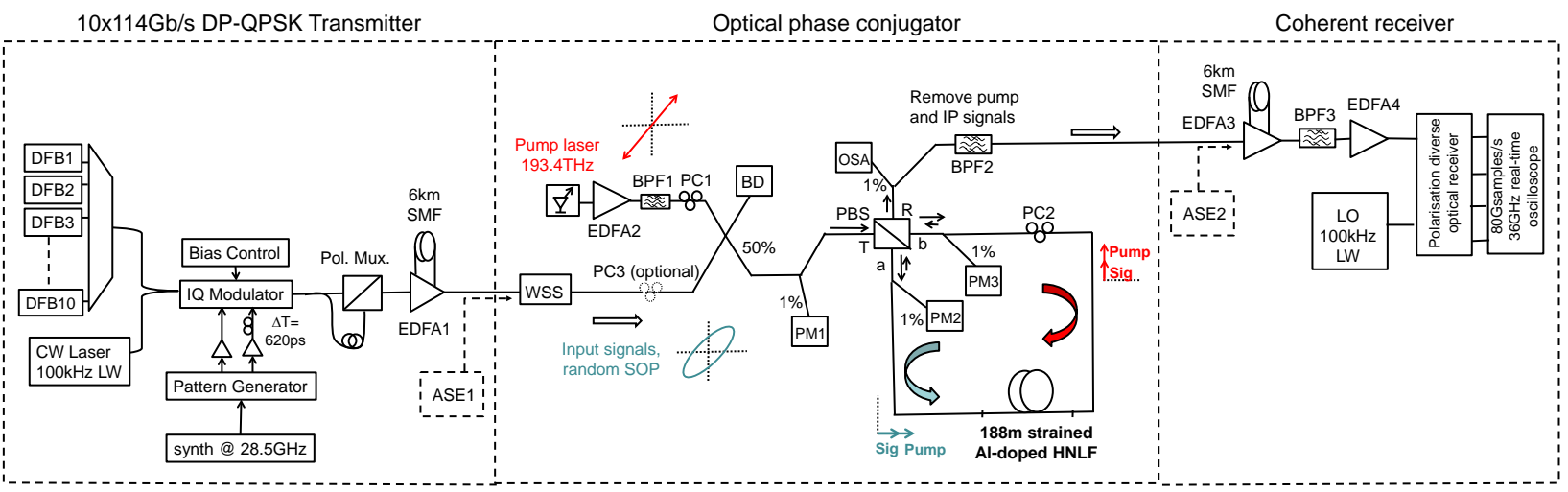

Figure 1: Experimental set-up of 1.14Tb/s DP-QPSK transmitter, conjugator and receiver

The experimental set-up is shown in Figure 1. Ten 100GHz-spaced DFB lasers ranging contiguously from $193.1 \mathrm{THz}$ $(1552.12 \mathrm{~nm})$ to $192.2 \mathrm{THz}(1559.79 \mathrm{~nm})$ are multiplexed together and combined with a tunable $100 \mathrm{kHz}$ linewidth laser to act as "signal under test" during the measurement cycle whereby the appropriate DFB is switched off. The WDM multiplex is QPSK modulated using normal and inverted 28.5Gbaud/s, $2^{31}-1$ patterns with an 18bit relative delay to drive an IQ modulator, followed by a PM emulator with 2ns delay between polarization states and optical amplification to $20 \mathrm{dBm}$ via EDFA1. The mid-stage of EDFA1 contains $6 \mathrm{~km}$ of SSMF to give a further $15 \mathrm{bit}$ decorrelation over the WDM band. A wavelength selective switch ( 5dB IL) is used to remove out-of-band ASE and a polarization controller (PC3) optionally fitted to explore OPC polarization dependence. Pump light derived 
from a $\sim 500 \mathrm{kHz}$ linewidth laser at $193.4 \mathrm{THz}(1550.1 \mathrm{~nm})$ is amplified (EDFA2) to $\sim 40 \mathrm{dBm}$, filtered by BPF1 (IL $\sim 2 \mathrm{~dB}$, 3dB BW $\sim 1 \mathrm{~nm}$ ) before combination with the signals via a $2 \times 250 \%$ coupler. The unused arm of the coupler is terminated at beam dump BD. The guard-band between pump and signals is set at 300GHz ( 2.4nm), and pump and signals coupled via the transmissive port $\mathrm{T}$ of a $2 \times 2$ polarization beam splitter (PBS) into a 188m bi-directional loop of HNLF optimized for the suppression of stimulated Brillouin scattering (SBS) [7]. The HNLF has a loss of 2.6dB and incorporates an Al-doped core and 100-1000g linear strain to obtain an SBS threshold of $\sim 1 W$. Nonlinear coefficient is 6.9 (W.km) $^{-1}$ and $\lambda_{0}$ ranges from $\sim 1552 \mathrm{~nm}$ at $100 \mathrm{~g}$ strain to $\sim 1564 \mathrm{~nm}$ at $1000 \mathrm{~g}$ strain. Pump polarization is controlled via PC1 to be as close to $45^{\circ}$ linear as possible by maximizing and equalizing the power measured on meters PM2 and PM3. No dithering of the pump signal is thus required for efficient OPC which is essential for minimization of performance penalties. Conjugation occurs when a pump component mixes with a signal component sharing the same polarization and direction around the loop to produce two sets of orthogonal conjugates per wavelength which are re-combined at the PBS, exiting at port R. The power and spectral shape of the conjugates at R is optimized using PC2 and ten conjugates produced from $193.7 \mathrm{THz}(1547.72 \mathrm{~nm})$ to $194.6 \mathrm{THz}$ (1540.56nm) which are filtered by BPF2. It should be noted that PC1 and PC2 are 'set-and-forget' polarization controllers and the setup can be left stably for many hours.

The receiver chain consists of amplifier EDFA3 containing 6km SSMF to un-disperse the conjugates, a $100 \mathrm{GHz}$ filter BPF4 followed by EDFA4 to provide $+4 \mathrm{dBm}$ into a polarization diverse coherent receiver. The conjugate and a local oscillator $(+10 \mathrm{dBm})$ are mixed in a $90^{\circ}$ optical hybrid before balanced detection and sampling via an $80 \mathrm{GS} / \mathrm{s}$, $36 \mathrm{GHz}$ scope. Data is processed off-line using standard Matlab ${ }^{\mathrm{TM}}$ DP-QPSK DSP, and performance characterized using $\mathrm{Q}^{2}(\mathrm{~dB})$ which is derived from the error vector magnitude and averaged over 10 x 57kSymbols.

\section{Experimental process and results}

Figures 2a) and 2b) show output spectra at port R for two settings of PC2 and for spectrally flat input signals. In 2a), PC2 is adjusted for maximum power per channel for the conjugates closest to the pump. In 2b), PC2 has been adjusted for overall conjugate flatness at the expense of conjugate power and spectral ripple on the original signals. This filtering effect is attributed to the Sagnac interferometric arrangement of the HNLF loop which alters the proportion of light (and hence OSNR) at particular wavelengths recombining through ports R and T of the PBS.

a)

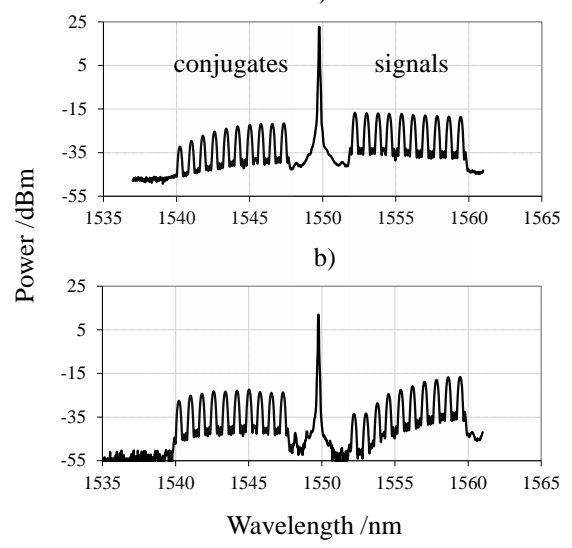

c)

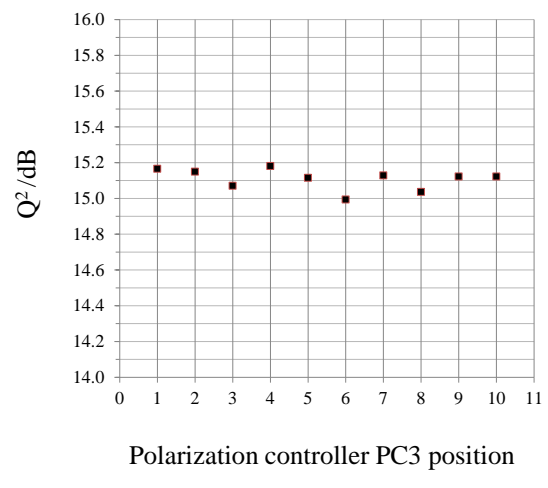

Figure 2: a) and b) show optical spectra at port R of PBS for two different loop polarizations after adjustment of PC2 c) Effect of input signal polarization on received $Q^{2}$ for ten different positions of PC3 for channel 193.7THz at 20dB OSNR

Optimum conjugate $\mathrm{Q}^{2}$ is obtained across all wavelengths for an input signal power of +3dBm per channel measured at PC3, while the maximum pump power $(+30 \mathrm{dBm})$ in each direction around the HNLF provides maximum conjugate OSNR. Detailed characterization measurements will be presented at the conference.

Figure 2c) illustrates the robustness of the conjugator to changes in input polarization via a systematic rotation of PC3. For each position the average $\mathrm{Q}^{2}$ of conjugate $193.7 \mathrm{THz}(1547.7 \mathrm{~nm})$ is plotted at 20dB OSNR - it can be seen that the variation in performance is small (std. dev. of $0.05 \mathrm{~dB}$ for this sample) and within natural fluctuation levels.

Figures $3 \mathrm{a}$ ) and $3 \mathrm{~b}$ ) illustrate the performance of the OPC as the OSNR is degraded by two different methods. In 3a), the OSNR is degraded post-OPC via noise source ASE2 and the effect on $\mathrm{Q}^{2}$ compared with back-to-back transmitter-receiver measurements for all ten $114 \mathrm{~Gb} / \mathrm{s}$ conjugates. In 3b), the noise is added pre-OPC via ASE1 to examine the OSNR degradation imparted by the conjugator - in this way, OPC conversion efficiency is estimated for different input OSNR conditions.

Figure 3a) shows that the WDM conjugation process imparts an OSNR-dependent $\mathrm{Q}^{2}$-penalty on the conjugates 
with respect to back-to-back, varying from a mean (across all wavelengths) of $\sim 0.1 \mathrm{~dB}$ (see inset of $3 \mathrm{a}$ ) to a mean of

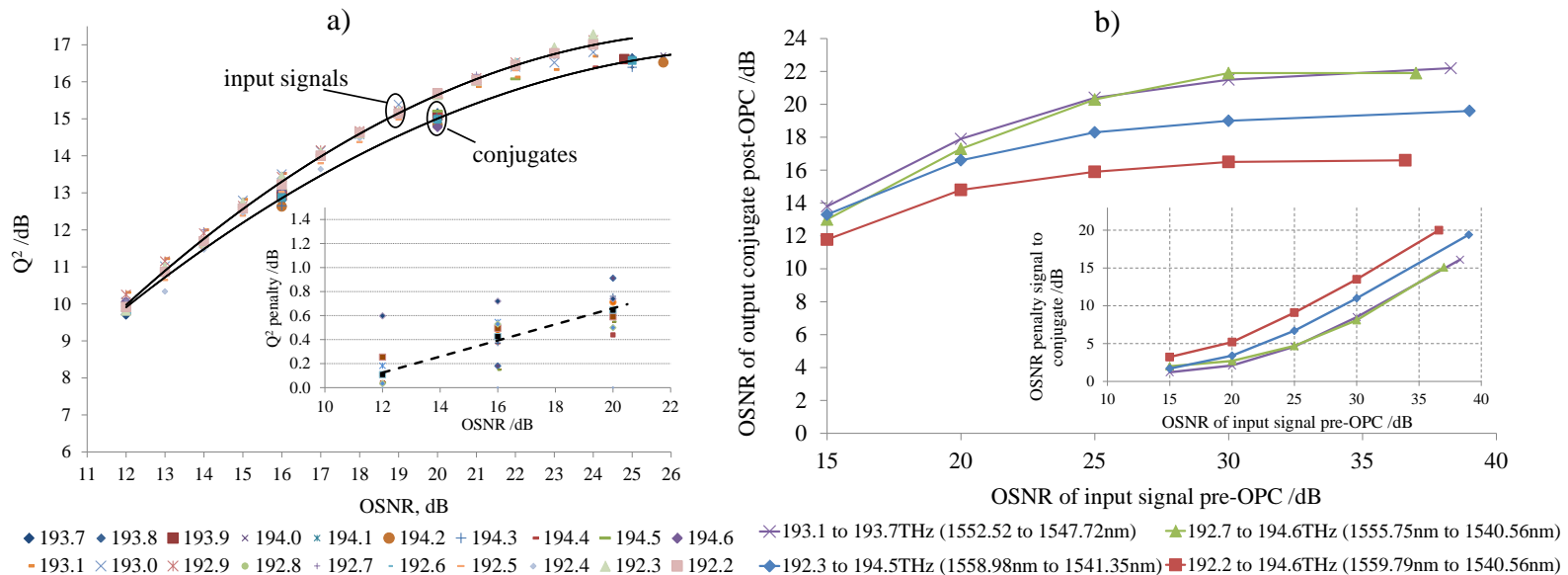

Figure 3: a) $\mathrm{Q}^{2}(\mathrm{~dB})$ vs OSNR ( $\mathrm{dB}$ ) for $10 \times 114 \mathrm{~Gb} / \mathrm{s}$ back-to-back (solid black line mean trendline) and respective conjugates (dashed black line mean trendline). Inset shows $\mathrm{Q}^{2}$ penalty at three OSNR values from same dataset b) Conjugate OSNR $(\mathrm{dB})$ post-OPC vs input signal OSNR (dB) pre-OPC. Inset shows OSNR penalty $(\mathrm{dB})$ vs input signal OSNR $(\mathrm{dB})$ for a sample of four conjugates across the band

$\sim 0.65 \mathrm{~dB}$ at 20dB OSNR. It is believed that this penalty derives from unwanted four wave mixing products appearing at conjugate wavelengths and from pump noise transfer and residual ASE. The reduced penalty at low OSNR offers potential for MSSI where the input OSNR to the conjugator will be low after transmission. The conjugate nearest the pump $(193.7 \mathrm{THz})$ consistently shows the largest penalty rising from $0.6 \mathrm{~dB}$ at $12 \mathrm{~dB}$ OSNR to $\sim 0.9 \mathrm{~dB}$ at $20 \mathrm{~dB}$. It is believed that this can be reduced by tighter ASE filtering after EDFA2.

The reduction in OSNR caused by conjugation is displayed in Figure 3b), and a plot of OSNR penalty vs input OSNR is shown in the inset for a subset of conjugates across the spectrum at fixed input signal and pump power. It can be seen that the OPC acts as a 'limiter' for high input OSNR, restricting maximum conjugate OSNR to $\sim 22 \mathrm{~dB}$ at $193.7 \mathrm{THz}$ for a high input OSNR of 38dB. Conversely for a degraded input OSNR of $15 \mathrm{~dB}$, this penalty is significantly reduced to a minimum of $1.2 \mathrm{~dB}$ for $193.7 \mathrm{THz}$ (conjugate nearest pump) and a maximum of $3.2 \mathrm{~dB}$ for $194.6 \mathrm{THz}$ (conjugate furthest from pump). It is expected that this will reduce further for lower input OSNR.

\section{Conclusions}

Polarization diverse optical phase conjugation of 10 x 114Gb/s 100GHz-spaced DP-QPSK signals with coherent detection is demonstrated and characterized for the first time using a strained HNLF loop. At $1 \mathrm{THz}(\sim 8 \mathrm{~nm})$ this represents a record spectral bandwidth for a fibre-based polarization diverse OPC band with $>1 \mathrm{~Tb} / \mathrm{s}$ capacity. An OSNR dependent $\mathrm{Q}^{2}$ penalty is observed rising from an average (across all conjugate wavelengths) of $0.1 \mathrm{~dB}$ at $12 \mathrm{~dB}$ OSNR to $0.65 \mathrm{~dB}$ at $20 \mathrm{~dB}$ OSNR. Maximum penalty observed at any wavelength or OSNR is $0.9 \mathrm{~dB}$ for the conjugate adjacent the pump. Variation of conjugate $Q^{2}$ with input signal polarization is shown to be small $( \pm 0.1 \mathrm{~dB})$ providing robust operation over several hours. OSNR penalty (in vs out) is characterized and shown to fall for all conjugate wavelengths as input OSNR reduces offering significant potential for mid-span spectral inversion applications.

\section{Acknowledgements}

This work was funded by the UK EPSRC under grants EP/J017582/1 (UNLOC) and EP/J009709/2.

\section{References}

[1] A Yariv et al, "Compensation for channel dispersion by nonlinear optical phase conjugation”, Optics Letters, Vol. 4, No. 2, February 1979. [2] D Nesset, MFC Stephens, AE Kelly, C Gilbertas, J Reed, KA Williams, S Bouchoule, R Kashyap, AD Ellis and DG Moodie, “40 Gbit/s transmission over $186.6 \mathrm{~km}$ of installed fibre using mid-span spectral inversion for dispersion compensation”, Vol.3, pp118-120, OFC 1999.

[3] SL Jansen, D van den Borne, PM Krummrich, S Spalter, GD Khoe and H de Waardt, "Long-Haul DWDM transmission systems employing optical phase conjugation”, IEEE Journal of Selected Topics in Quantum Electronics, Vol. 12, No. 4, pp.505-520, July/August 2006.

[4] S. Watanabe, S. Takeda, and T. Chikama, "Interband wavelength conversion of $320 \mathrm{~Gb} / \mathrm{s}(32 \times 10 \mathrm{~Gb} / \mathrm{s})$ WDM signal using a polarization insensitive fiber four-wave mixer,” in Proc. ECOC’98, 1998, pp. 85-87.

[5] P. Minzioni et al, "Optical Phase Conjugation for Dispersion and Nonlinearity Compensation in a 1600km, 42 Gb/s Quasi-Lossless System," Advances in Optical Sciences Congress, OSA Technical Digest NThA5

[6] Monir Morshed, Liang B. Du, Benjamin Foo, Mark D. Pelusi, and A. J. Lowery, “Optical Phase Conjugation for Nonlinearity Compensation of 1.21-Tb/s Pol-Mux Coherent Optical OFDM”, OECC 2013, Kyoto, Japan, July 2013.

[7] Lars Grüner-Nielsen et al, “A silica based highly nonlinear fibre with improved threshold for stimulated Brillouin scattering”, paper Tu.4.D3, ECOC 2010, 19-23 September 2010, Torino, Italy. 